\title{
Tourisme et emplois : singularités, permanences, transformations
}

\section{Christophe Guibert, Jean Lagueux et Nathalie Montargot}

\section{(2) OpenEdition}

\section{Journals}

Édition électronique

URL : http://journals.openedition.org/tourisme/2309

DOI : 10.4000/tourisme.2309

ISSN : 2492-7503

Éditeur

Éditions touristiques européennes

Référence électronique

Christophe Guibert, Jean Lagueux et Nathalie Montargot, «Tourisme et emplois : singularités, permanences, transformations », Mondes du Tourisme [En ligne], 16 | 2019, mis en ligne le 01 décembre 2019, consulté le 24 septembre 2020. URL : http://journals.openedition.org/tourisme/2309 ; DOI : https://doi.org/10.4000/tourisme.2309

Ce document a été généré automatiquement le 24 septembre 2020

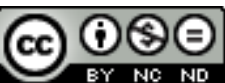

Mondes du tourisme est mis à disposition selon les termes de la licence Creative Commons Attribution - Pas d'Utilisation Commerciale - Pas de Modification 4.0 International. 


\title{
Tourisme et emplois : singularités, permanences, transformations
}

\author{
Christophe Guibert, Jean Lagueux et Nathalie Montargot
}

1 Les pratiques et les destinations touristiques se diffusent dans un nombre toujours plus grand de lieux et de pays. La diversification des manières de "faire du tourisme » constitue également une caractéristique contemporaine. Pour autant, à chaque étape, chaque niveau du «système touristique » (MIT, 2005), les fonctions exercées par les professionnels, bien qu'en mutation, sont centrales afin de proposer une offre de services touristiques en cohérence avec les attendus et exigences des touristes euxmêmes. Activité marchande, le tourisme présuppose donc, de l'acte de réservation (d'un séjour, d'une chambre d'hôtel, d'un cours de parapente par exemple) à la prestation (visite d'un lieu patrimonial ou d'une cave viticole, pratique d'une activité sportive par exemple), tout un enchevêtrement d'activités professionnelles nécessitant des compétences et des savoir-être propres aux offres de services de type touristique. Si les tâches et fonctions des emplois touristiques sont variées au sein d'un «espace pluriel» (Guillaud, 2018), renforçant dès lors le piège de la charge sémantique et nominale que peut revêtir clandestinement ce terme, les rémunérations sont d'une part assez hétérogènes (allant $d u$ salaire minimum à plus du double du salaire minimum, y compris pourboires et autres gratifications) et les niveaux de qualifications sont, d'autre part, également très disparates (du CAP au Bac +5 en France par exemple). Les transformations économiques, technologiques, culturelles et sociétales qui peuvent être identifiées dans la plupart des secteurs professionnels affectent également le champ du tourisme. Partant, plusieurs thématiques, engageant autant de questions, peuvent être identifiées - sans que celles-ci ne soient exhaustives.

\section{Effet structurant du « numérique »}

2 La multiplication des procédures, plus ou moins formalisées, liées au développement d'Internet, telle que la réservation «en ligne» de produits touristiques (séjours, circuits, hébergements, etc.), ont des effets qualitatifs et quantitatifs sur les métiers 
«historiques" du secteur touristique. De "nouveaux » métiers, de nouvelles tâches professionnelles émergent. Comment le déploiement des nouvelles technologies dans des plans stratégiques digitaux est-il accepté et intégré sur le terrain par le personnel ? Comment celui-ci perçoit-il l'évolution de ses fonctions, de son métier? (Sotiriadis et Varvaressos, 2016). Comment les voyagistes ont-ils opéré des transformations sociales (p. ex. rationalisation des effectifs salariés en agence de voyage) ? Quelles ont été les stratégies de positionnement et/ou de repositionnement stratégique des entreprises du secteur face à la concurrence internationalisée ? Quelle forme prend l'offre touristique lorsque les individus ne sont plus au centre de la prestation de service (services touristiques en ligne comme les visites guidées en baladodiffusion (podcast), service de conciergerie virtuel, etc.)?

\section{Visibilité/invisibilité}

Si les métiers du tourisme sont, dans les représentations spontanées, identifiables aux personnels d'accueil (dans les offices de tourisme, dans les hôtels, campings et résidences, au sein des sites touristiques patrimoniaux, etc.) et de service (moniteurs d'activité sportive - ski, surf, voile, etc. -, guide conférencier, animateur de colonie, etc.), il existe de nombreuses professions éloignées physiquement et symboliquement du contact direct avec les clientèles touristiques. Les personnels de service (Pinna, 2013) tels que les "femmes de chambre » ou les plongeurs, dans la restauration en particulier, bien qu'invisibles (Castel, 1999) aux yeux des touristes ou circonscrits à de courtes et rares interactions, sont néanmoins primordiaux et nécessaires dans le « bon fonctionnement » d'une offre touristique. De surcroitt, de plus en plus d'emplois reliés au tourisme le sont en arrière-scène (back office) mais ajoutent beaucoup de valeur à la prestation de service. Quelles sont, dès lors, les formes de reconnaissance pour les acteurs de part et d'autre des frontières de l'accueil ?

\section{Des professionnels à contre-espace et contretemps}

Le temps des vacances, des congés est caractérisé par une forte concentration, hivernale et estivale surtout, à la fois spatiale (les stations de ski, les stations balnéaires) et temporelle. Or, être salarié (ou à son compte) d'une entreprise ou d'une association de services touristiques, c'est travailler à contretemps et contre-espace, autrement dit hors des lieux et temporalités des cadres de vie ordinaires des touristes (Sébileau, 2014). Comment, toutefois, conjuguer activité professionnelle estivale lors des congés scolaires et vie familiale ? Comment pérenniser une vie conjugale lorsqu'on fait « les saisons » (en été à la plage comme moniteur de voile, l'hiver comme guide de haute montagne)? Quelles solutions collectives (telles que les groupements d'employeurs dans certaines régions) sont inventées pour faciliter l'emploi des saisonniers et les fidéliser? Pour quels résultats? De quelles façons le tourisme peut-il surmonter ces contraintes temporelles sectorielles propres pour rendre l'emploi touristique globalement plus attractif? 


\section{Conjuguer passion et activité professionnelle}

5 En lien avec la thématique précédente, de nombreux travaux ont montré que l'engagement sur le modèle de la passion, dans certains secteurs professionnels, constituait une spécificité que l'on peut adosser au secteur touristique. Passionné de "vieilles pierres", passionné de surf (Guibert et Slimani, 2011) ou d'équitation, des professionnels du tourisme s'engagent dans des carrières à l'aune de leurs propres pratiques personnelles, de leurs passions. Comment ces professionnels négocient-ils la place de leur passion dans leur activité professionnelle? Si l'on considère l'envers du décor, peut-on parler de désenchantement, voire de désillusion? Les emplois touristiques font-ils toujours partie d'un projet de carrière ou bien s'agit-il seulement d'un passage avant de trouver un emploi plus « prometteur »?

\section{Une relation d'emploi spécifique?}

6 En dehors de la possible conjugaison passion/activité professionnelle, quelle est la nature de la relation d'emploi des différents professionnels du tourisme, acteurs de ce secteur par choix ou par contrainte? Quelles sont ses éventuelles spécificités, ses différentes formes eu égard au niveau de qualification des emplois? Comment peut-elle être appréhendée dans le cadre d'une théorie de l'échange social renouvelée (Cropanzano et al., 2017) afin d'aider à prévoir les comportements au travail au-delà d'un simple décryptage a posteriori et de susciter les comportements désirables pour des organisations du secteur touristique confrontées à de nouvelles exigences (hôtellerie life style, approche narrative, contact enrichi personnalisé, etc.) ? Comment s'articulent les comportements positifs et négatifs des professionnels du tourisme confrontés à des expériences de travail variées et parfois imprévisibles (engagement/retrait, comportements citoyens/comportements contreproductifs, confiance/défiance, jeu émotionnel en profondeur/jeu émotionnel en surface, etc.)? Quel est le rôle des partenaires sociaux, souvent peu présents, dans la définition des contrats sociaux?

\section{Les stratégies $\mathrm{RH}$ : permanences ou révolution(s)?}

7 En appui des thématiques précédentes et prenant en compte les effets conjoncturels de la crise économique qui touche, quoique différemment, les pays européens et nordaméricains, comment se définissent ou se redéfinissent les stratégies entreprises en termes de "politique RH»? Quelles articulations sont possibles entre les stratégies RH corporate et leur déclinaison sur le terrain? La croissance touristique mondiale met une pression sur l'offre touristique, qui à son tour exerce une pression sur la demande de main-d'œuvre. Cette même main-d'œuvre est également sollicitée par d'autres secteurs d'activité, souvent plus attrayants pour l'employé. Quelles sont les nouvelles stratégies utilisées par les entreprises touristiques pour accéder à de nouveaux bassins de travailleurs et pour retenir ces derniers? Enfin, les formations proposées (en présentiel ou en e-learning) sont-elles en adéquation avec les emplois et les compétences recherchés par les organisations touristiques? Quelle place est accordée aux soft skills dans les programmes de formation et dans les politiques de recrutement? 
8 Pour répondre à ces diverses problématisations, sept articles mobilisant des cadres disciplinaires variés (droit, histoire, géographie, sciences de gestion) et des terrains internationaux spécifiques abordent la question de l'emploi mais aussi des formations. L'article de Philippe Violier, intitulé "Statistiques et emploi », propose une critique argumentée des modalités de production et des usages des données statistiques spécifiques à «l'emploi touristique », pointant les enjeux de définition dont sont issues les fausses évidences et les erreurs interprétatives. Le texte proposé par Julie Manfredini, «Faire d'une passion une profession, la place des syndicats d'initiative dans le tourisme français ", interroge, sous l'angle de l'histoire, la création progressive de métiers singuliers au sein des «syndicats d'initiative " à la fin du xix ${ }^{e}$ siècle. Les convictions des élites culturelles locales ont contribué à structurer la professionnalisation des premières associations de promotion touristique. Louis Jolin analyse, du point de vue du droit, "Le cadre juridique de travail des employés de service du secteur de la restauration. La question spécifique du salaire ». Un parallèle est proposé entre les situations des «employés à pourboire » au Québec et en France. Jérôme Piriou et Marie-Noëlle Rimaud analysent quant à eux les effets des transformations institutionnelles à l'œuvre en France à l'aune d'une comparaison localisée. Leur article, «Réformes territoriales et exercice de la compétence tourisme par l'institution publique départementale française. Cas exploratoire de la CharenteMaritime et des Deux-Sèvres", analyse spécifiquement l'échelon des comités départementaux du tourisme et les assignations de compétences qui leur sont liées. Jean Lagueux et Audrey Nanot, dans un article intitulé « Engagement des étudiants en tourisme, hôtellerie et restauration envers leur domaine d'études et leur carrière : les facteurs déterminants", questionnent les motivations et les prédispositions des étudiants à s'engager dans des formations diplômantes au Canada. À partir d'une enquête quantitative, ils montrent que les expériences et les satisfactions des étudiants lors d'expériences professionnelles passées sont centrales pour saisir leur niveau d'investissement dans leurs formations respectives. Boualem Kadri, Mohamed Reda Khomsi et Cyril Martin proposent une étude relative à "L'évolution du discours sur les ressources humaines en tourisme: entre théories et pratiques managériales». Cet article interroge, en particulier à partir d'un corpus de la revue Espaces, tourisme et loisirs, la prise en compte du tourisme comme objet de recherche scientifique, ainsi que la construction sociale des discours et des théories qui lui sont liés. Enfin, Bertrand Dongmo Temgoua, dans l'article intitulé «Quelles ressources humaines pour le tourisme de mémoire? Le profil des agents prestataires de services lors des funérailles bamilékés du Cameroun ", analyse l'adéquation entre le profil des acteurs du tourisme et leur formation. Au-delà de la qualification initiale, l'efficacité de ces agents dépend également de compétences et de connaissances singulières, telles que les conventions sociales attachées au phénomène des funérailles bamilékés. 


\section{BIBLIOGRAPHIE}

Robert CASTEL, Les métamorphoses de la question sociale, Folio Essais, 1999.

Russel CROPANZANO, Erica ANTHONY, shanna DANIELS, Alison HALL, « Social exchange theory : A critical review with theoretical remedies », The Academy of Management Annals, $\mathrm{n}^{\circ}$ 11(1), 2017.

Équipe MIT, Tourismes 2. Moments de lieux, « Mappemonde », Belin, 2005.

Christophe GUIBERT et Hassen SLIMANI, Emplois sportifs et saisonnalités. L'économie des activités nautiques : enjeux de cohésion sociale, L'Harmattan, 2011.

Étienne GUILLAUD, De l'attrait à l'usure. Les trajectoires professionnelles des éducateurs sportifs en nautisme, thèse de doctorat en sociologie, Université de Nantes, 2018.

Gabriele PINNA, «Vendre du luxe au rabais : une étude de cas dans l'hôtellerie haut de gamme à Paris ", Travail et emploi, $\mathrm{n}^{\circ}$ 136, 2013.

Arnaud SÉBILEAU, « Rester après la saison : l'économie symbolique du néoruralisme balnéaire », Juristourisme, $\mathrm{n}^{\circ}$ 163, 2014.

Marios SOTIRIADIS et Stelios Varvaressos, «Crucial Role and Contribution of Human Resources in the Context of Tourism Experiences : Need for Experiential Intelligence and Skills », dans Marios SOTIRIADIS et Dogan GURSOY (dir.), The Handbook of Managing and Marketing Tourism Experiences, Emerald Group Publishing Limited, 2016.

\section{AUTEURS}

\section{CHRISTOPHE GUIBERT}

Maître de conférences en sociologie, Université d'Angers, UFR ESTHUA Tourisme et culture, Laboratoire ESO (UMR CNRS 6590), christophe.guibert@univ-angers.fr

\section{JEAN LAGUEUX}

Sciences de gestion, ESG UQAM, lagueux.jean@uqam.ca

\section{NATHALIE MONTARGOT}

Professeure associée, docteur en sciences de gestion, La Rochelle Business School - Excelia Group, CEREGE (EA1722), montargotn@excelia-group.com 